\title{
Knockdown of ubiquitin associated protein 2-like inhibits the growth and migration of prostate cancer cells
}

\author{
DONG LI and YIRAN HUANG \\ Department of Urology, The Affiliated Renji Hospital of the Medical College, \\ Shanghai Jiaotong University, Shanghai 200127, P.R. China \\ Received April 22, 2014; Accepted June 18, 2014
}

DOI: $10.3892 /$ or.2014.3360

\begin{abstract}
The ubiquitin-proteasome system (UPS) is involved in the development of cancer in various functions. Inhibition of the ubiquitin-proteasome has been revealed to be a powerful therapeutic method for carcinoma. Ubiquitin associated protein 2-like (UBAP2L) is believed to be involved in the ubiquitin-proteasome pathway; however, the role of UBAP2L in human prostate cancer is still unknown. In the present study, we found that UBAP2L was expressed in a number of prostate carcinoma cell lines. Using lentiviral-mediated RNA interference (RNAi), we efficiently knocked down endogenous UBAP2L expression at the mRNA and protein levels. After UBAP2L disruption, the proliferation and colony formation ability were significantly reduced in the PC-3 and DU145 cells. Flow cytometric analysis showed that UBAP2L knockdown blocked cell cycle progression. Downregulation of UBAP2L inhibited the migration of PC-3 and DU145 cells, as determined by Transwell assay. Moreover, depletion of UBAP2L blocked the AMPK $\alpha$, Bad and PRAS40 signaling pathways. In conclusion, our results suggest that UBAP2L may play a role in prostate cancer growth and metastasis, and knockdown of UBAP2L by RNAi may serve as a potential therapeutic approach for prostate cancer.
\end{abstract}

\section{Introduction}

Prostate cancer, formed in the gland of the male reproductive system, is one of the leading causes of cancer-related death in males. It is more common in developed countries compared with developing countries (1). According to an NIH report, more than two million men in the United States are diagnosed with prostate cancer. Clinical surgery, radiation therapy,

Correspondence to: Professor Yiran Huang, Department of Urology, The Affiliated Renji Hospital of the Medical College, Shanghai Jiaotong University, 1630 Dongfang Road, Shanghai 200127, P.R. China E-mail: yiranhuangdr@163.com

Key words: UBAP2L, prostate cancer, RNA interference, proliferation, migration chemotherapy and numerous other therapeutic methods are used for the treatment of prostate cancer. However, prostate cancer is still the second leading cause of cancer-related death in men in the United States, and more than 29,000 men died of prostate cancer in 2013. More efficient and specific therapeutic methods are needed to be developed. Lentiviral-based gene therapeutic methods have been approved more than a decade ago and no apparent serious side effects have been reported (2).

The ubiquitin-proteasome pathway is a common pathway for protein degradation in eukaryotes. It is involved in a wide variety of cellular processes including apoptosis, cell cycle control, cell adhesion and tumor growth (3). Inhibition of the ubiquitin-proteasome pathway has emerged as a powerful strategy for anticancer therapy (4-6). Several proteasome inhibitors have been approved for the treatment of cancer clinically $(4,5,7,8)$. Ubiquitin associated protein 2-like (UBAP2L) was found to be cofractionated with ubiquitin in the highdensity fraction and it is colocalized in the ubiquitin-containing aggregates after proteasome inhibition (9), which suggests that UBAP2L may be involved in the ubiquitin-proteasome pathway. However, the function of UBAP2L in prostate cancer is largely unknown.

In the present study, we assessed the expression of UBAP2L in different human prostate carcinoma cell lines. Using lentiviral-mediated RNAi, we successfully depleted endogenous UBAP2L expression. The effects of UBAP2L inhibition on prostate carcinoma cell proliferation, tumorigenesis and metastasis were then investigated.

\section{Materials and methods}

Cell culture. Human prostate carcinoma cell lines, DU145, PC-3, LN-Cap, 22RV1, and human embryonic kidney cell line 293T (HEK293T) were obtained from the Cell Bank of the Chinese Academy of Science (Shanghai, China). PC-3 cells were cultured in Ham's/F-12 medium (Gibco-BRL) supplemented with $10 \%$ fetal bovine serum (FBS). LN-Cap and 22RV1 cells were cultured in ATCC-formulated RPMI-1640 supplemented with $10 \%$ FBS. DU145 cells were cultured in Ham's/F-12 medium supplemented with 10\% FBS and 1\% non-essential amino acids (NEAA). HEK293T cells were cultured in DMEM supplemented with $10 \%$ FBS. Cells were cultured in a $5 \% \mathrm{CO}_{2}$ incubator at $37^{\circ} \mathrm{C}$. 
Lentivirus packaging and infection. Short hairpin RNA (shRNA) (5'-GCCAATACTGATGATAACTATCTCGAGA TAGTTATCATCAGTATTGGCTTTTT-3') was designed to knock down UBAP2L (NM_001127320.1). The non-targeting nucleotide sequence (5'-CTAGCCCGGTTCTCCGAAC GTGTCACGTATCTCGAGATACGTGACACGTTCGGAGA ATTTTTTTAAT-3') was designed as the control. The shRNA oligos were ligated into the lentivirus expression plasmid pFH-L (Shanghai Hollybio, China). To package the shUBAP2L and shCon lentivirus, shRNA plasmids, envelope plasmid pVSVG-I and packaging plasmid pCMV $\Delta$ R8.92 (Shanghai Hollybio, China) were transfected into HEK293T cells using Lipofectamine 2000 (Invitrogen) according to the manufacturer's instructions. Three days after transfection, the lentivirus-containing media were collected and ultracentrifuged for infection. PC-3 and DU145 cells were cultured in 6-well plates at a density of 50,000 cells/well, respectively. The lentivirus was added to the culture medium at an MOI of 40. Three days after infection, the cells were observed under a microscope (10x objective lens), and GFP-positive cell numbers were counted to calculate the infection efficiency.

Real-time $q R T-P C R$. After being washed by ice-cold PBS, total-RNA was extracted using TRIzol reagent (Invitrogen) following the manufacturer's instructions. M1705 M-MLV reverse transcriptase kit (Promega) was used to synthesize cDNA. Real-time qRT-PCR was performed to evaluate the expression level of UBAP2L, and $\beta$-actin was used as an endogenous control. The primers used were UBAP2L, forward, 5'-ACACAATCCCCATCACTGGT-3' and reverse, 5'-CAGAGGAGAAGACGGAGGTG-3'; $\beta$-actin, forward, 5'-GTGGACATCCGCAAAGAC-3' and reverse, 5'-AAAGGG TGTAACGCAACTA-3'. Relative mRNA was determined by the formula $2^{-\Delta \Delta \mathrm{Ct}}(\mathrm{Ct}$, cycle threshold).

Western blot analysis and Pathscan ${ }^{\circledR}$ Intracellular Signaling Array. Five days after infection, prostate carcinoma cells were washed twice with ice-cold PBS and lysed in 2X SDS sample buffer (10 mM EDTA, 4\% SDS, $10 \%$ glycine in $100 \mathrm{mM}$ Tris- $\mathrm{HCl}$ buffer, $\mathrm{pH}$ 6.8) for $1 \mathrm{~h}$ at $4^{\circ} \mathrm{C}$. Total cell lysates were then centrifuged $\left(12,000 \mathrm{rpm}, 15 \mathrm{~min}, 4^{\circ} \mathrm{C}\right)$, and the supernatants were employed for further processing. The protein concentration was determined using the BCA protein assay kit. Equal amounts of proteins $(30 \mu \mathrm{g})$ were loaded and separated on $10 \%$ SDS-PAGE gels and transferred onto PVDF membranes (Millipore). Proteins were probed overnight at $4^{\circ} \mathrm{C}$ with the primary antibodies UBAP2L (1:2,000; Abcam; cat. ab70319) or GAPDH (1:50,000; Proteintech Group, Inc.; cat. 10494-1-AP), followed by incubation with horseradish peroxidase-conjugated goat anti-rabbit $\operatorname{IgG}(1: 5,000$; Santa Cruz; cat. sc-2054) or goat anti-mouse IgG (1:5,000; Santa Cruz; cat. sc-2005) at room temperature for $2 \mathrm{~h}$. GAPDH was used as the internal standard.

To detect the activation of intracellular signaling, Pathscan Intracellular Signaling Array was performed. In brief, 5 days after lentiviral infection, DU145 cells were collected and lysed. Intracellular signaling was detected using the Pathscan Intracellular Signaling Array Kit (Cell Signaling Technology) following the manufacturer's instructions.
MTT assay. After lentiviral infection for 4 days, PC-3 and DU145 cells were seeded in 96-well plates at a density of 2,000 and 2,500 cells/well, respectively. At different time points, $20 \mu \mathrm{l}$ of $5 \mathrm{mg} / \mathrm{ml}$ MTT [3-(4, 5-dimethylthiazol-2-yl)-2, 5-diphenyltetrazolium bromide] was added into each well. After incubation with MTT for $3 \mathrm{~h}, 100 \mu \mathrm{l}$ of acidic isopropanol (10\% SDS, 5\% isopropanol, $0.01 \mathrm{~mol} / 1 \mathrm{HCI})$ was added. The formazan precipitate was then dissolved by shaking for $10 \mathrm{~min}$. The absorbance of each well was recorded at a wavelength of $595 \mathrm{~nm}$ using a microplate reader.

Colony formation assay. After lentiviral infection for 4 days, PC-3 and DU145 cells were seeded into 6-well plates at an initial density of 400 and 500 cells/well, respectively. The medium was changed at three-day intervals. PC-3 and DU145 cells were washed with PBS and fixed with $4 \%$ paraformaldehyde for $30 \mathrm{~min}$ at room temperature after culture for 13 and 8 days, respectively. The fixed cells were then stained with freshly prepared diluted crystal violet for $10 \mathrm{~min}$, washed with water and air-dried. The total number of colonies was counted using a light microscope.

Cell cycle analysis. After lentiviral infection for 4 days, PC-3 and DU145 cells were seeded into $6-\mathrm{cm}$ dishes at a density of 200,000 cells/dish and cultured until the confluency of the cells reached $\sim 80 \%$. Cells were harvested, washed with icecold PBS and fixed overnight at $75 \%$ ethanol at $4{ }^{\circ} \mathrm{C}$. Propidium iodide (PI) staining was performed following the instructions provided in the kit (C1052; Biyuntian). The fluorescence of PI in the cells was measured using Cell Lab Quanta (Beckman Coulter).

Transwell migration assay. In brief, 3 days after infection, 70,000 PC-3 or 50,000 DU145 cells were seeded into the upper chamber of Transwell plates $(8.0-\mu \mathrm{m}$ pore; Corning Costar) in $200 \mu \mathrm{l}$ of serum-free medium, and $500 \mu \mathrm{l}$ of medium containing $10 \%$ FBS was added to the lower chamber as a chemoattractant. After incubation for $24 \mathrm{~h}$, cells on the top surface of the membrane were removed. After fixation, cells that migrated to the bottom surface of the membrane were stained using crystal violet. Images were captured under a light microscope (10x objective lens), and cell numbers were counted.

Statistical analysis. All data are presented as the mean \pm SD of three independent experiments performed in triplicate. Statistical analysis was performed based on a Student's t-test. $\mathrm{P}<0.05$ was considered to indicate a statistically significant difference.

\section{Results}

UBAP2L expression in prostate carcinoma cell lines. Expression levels of UBAP2L in prostate carcinoma cells were analyzed by qRT-PCR and western blotting. As shown in Fig. 1A, mRNA levels of UBAP2L were detected in all 4 prostate carcinoma cell lines: DU145, PC-3, LN-Cap and 22RV1. We found a strong immunoreactive band of UBAP2L in the samples of DU145, PC-3 and LN-Cap cells, while a very faint band was noted in the sample of 22RV1 cells (Fig. 1B). 
A

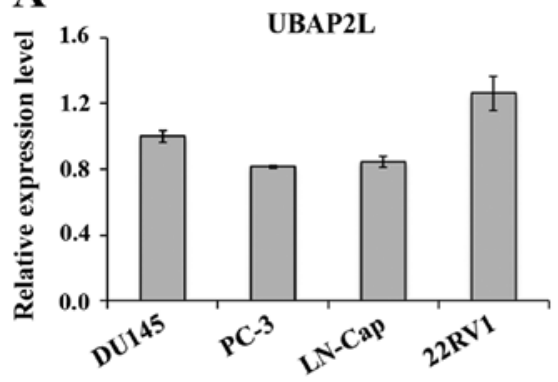

B

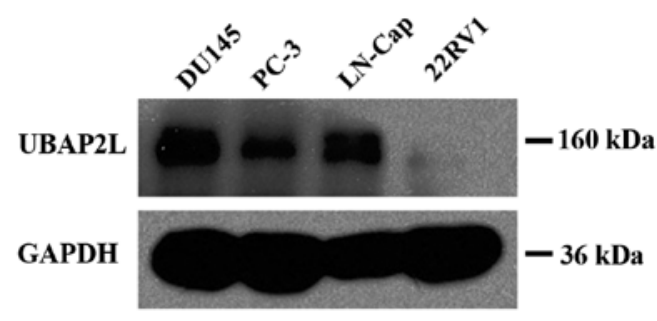

Figure 1. Expression analysis of UBAP2L in prostate carcinoma cells. (A) qRT-PCR analysis of the UBAP2L mRNA levels in the prostate cancer cell lines DU145, PC-3, LN-Cap and 22RV1. $\beta$-actin was used as an internal control. Data represent the mean \pm SD of three independent experiments. (B) Western blot analysis of the UBAP2L protein levels in the prostate cancer cell lines DU145, PC-3, LN-Cap and 22RV1. GAPDH was used as a loading control. Data represent one out of three separate experiments.

A

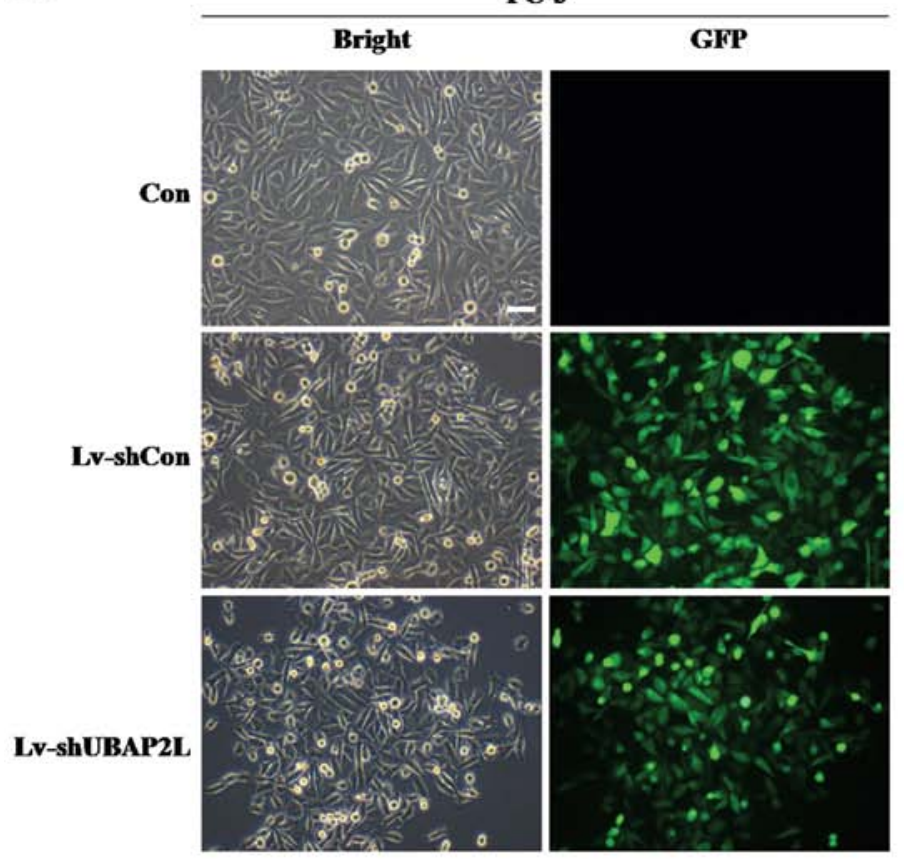

B

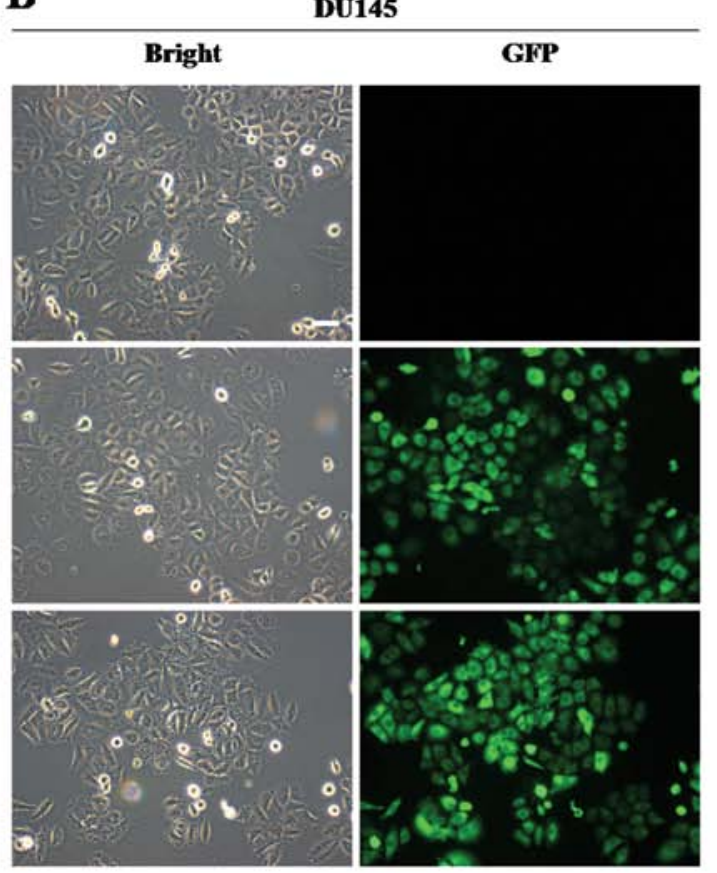

Figure 2. Infection efficiency of the lentivirus in (A) PC-3 and (B) DU145 cells. Images were captured by fluorescence microscopy, and GFP-positive cells were counted. Infection efficiency was evaluated by monitoring GFP expression. Scale bar, $50 \mu \mathrm{m}$.

Therefore, PC-3 and DU145 cells were used for the following loss-of-function investigation.

The lentivirus efficiently infects PC-3 and DU145 cells. To investigate the function of UBAP2L in prostate carcinoma cells, we took advantage of lentiviral-mediated RNAi technology. Firstly, the infection efficiency of the lentivirus was evaluated. Four days after lentiviral infection, GFP was robustly expressed in the PC-3 and DU145 cells (Fig. 2A and B). More than $90 \%$ cells were infected by the recombinant lentivirus.

Lv-shUBAP2L infection efficiently reduces endogenous $U B A P 2 L$ expression. Four days after lentiviral infection, the expression levels of UBAP2L in the PC-3 and DU145 cells were measured. Using qRT-PCR, we found that the relative expression of UBAP2L was markedly decreased after Lv-shUBAP2L infection, compared with the control and the non-infected groups (Fig. 3A and C). The protein levels of UBAP2L in the PC-3 and DU145 cells were also reduced after LV-shUBAP2L infection, as measured by western blotting (Fig. 3B and D).

Knockdown of UBAP2L suppresses cell proliferation and colony formation. To investigate whether UBAP $2 \mathrm{~L}$ is involved in the proliferation and tumorigenesis of prostate carcinoma cells, MTT and colony formation assays were performed. As shown by cell growth curve, compared with the Lv-shCon-infected cells, the proliferation of Lv-shUBAP2L-infected cells was significantly decreased. A $38.0 \%$ reduction in the $\mathrm{PC}-3$ cells (Fig. $4 \mathrm{~A}$ ) and a $49.8 \%$ reduction in the DU145 cells were noted (Fig. 4B).

Colony formation assay was then carried out. As shown in Fig. 5, the numbers of colonies formed in the Lv-shUBAP2L groups (52.0 \pm 5.3 PC-3 cells and 177.7 \pm 4.2 DU145 cells) were 

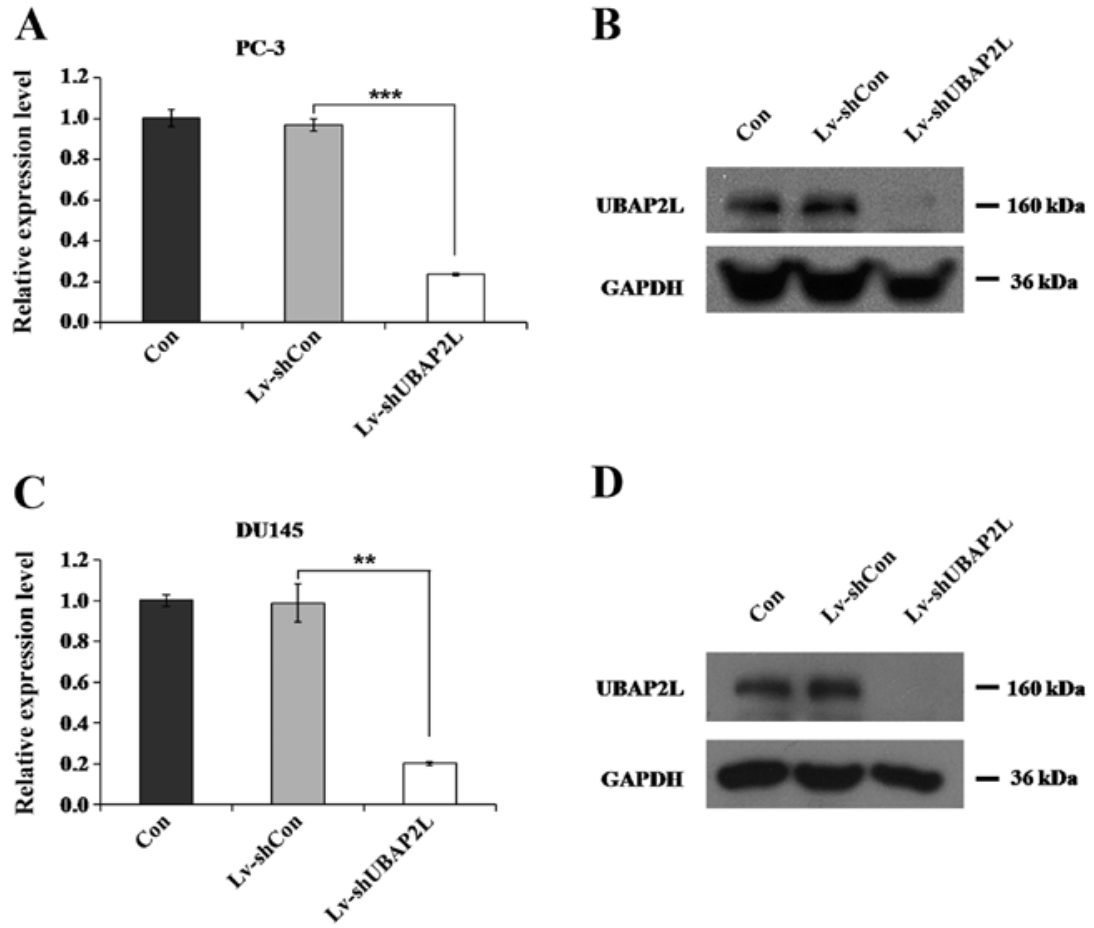

D

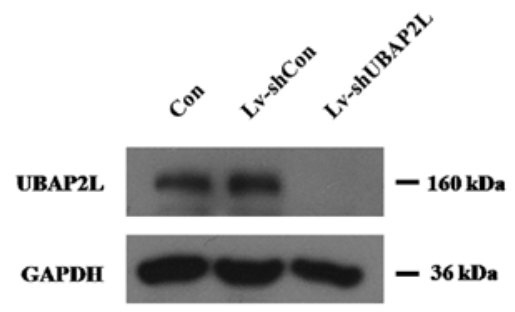

Figure 3. UBAP2L expression in PC-3 and DU145 cells is inhibited by lentiviral-mediated shRNA. qRT-PCR analysis of the UBAP2L mRNA levels in (A) PC-3 and (C) DU145 cells after lentiviral infection. $\beta$-actin was used as an internal control. Data represent the mean \pm SD of three independent experiments. ${ }^{* *} \mathrm{P}<0.01,{ }^{* * *} \mathrm{P}<0.001$, compared with Lv-shCon. Western blot analysis of the UBAP2L protein levels in (B) PC-3 and (D) DU145 cells after lentiviral infection. GAPDH was used as a loading control. Data represent one out of three separate experiments.

$\mathbf{A}$

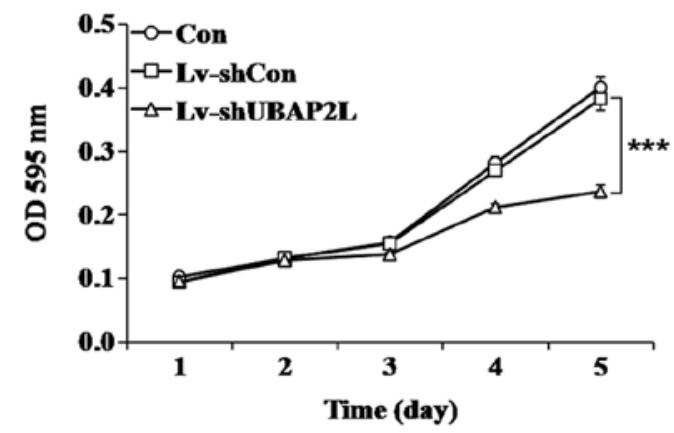

B

DU145

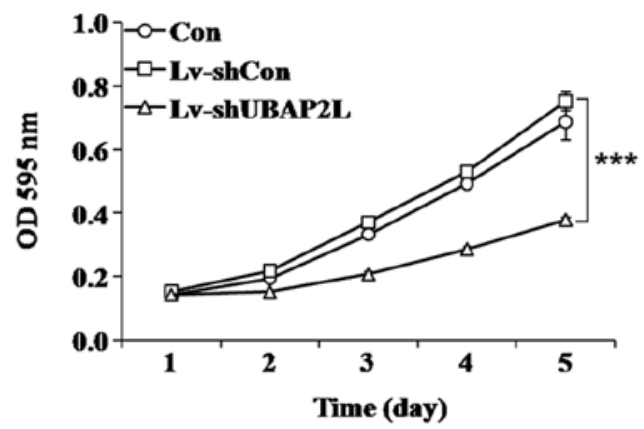

Figure 4. Knockdown of UBAP2L inhibits the proliferation of prostate carcinoma cells. Growth curves of (A) PC-3 and (B) DU145 cells were generated by MTT assay. The proliferation rate was reduced after $\mathrm{Lv}$-shUBAP2L infection. Data represent the mean $\pm \mathrm{SD}$ of three independent experiments. ${ }^{* * *} \mathrm{P}<0.001$, compared with Lv-shCon.

markedly lower than the numbers of colonies in the Lv-shCon groups (159.7 \pm 6.0 PC-3 cells and $254.7 \pm 9.1$ DU145 cells). There was no significant difference in the number of colonies between the Lv-shCon groups and the non-infected groups. These results suggest that UBAP2L may play an important role in the tumorigenesis of prostate carcinoma.

Knockdown of UBAP2L leads to cell cycle arrest of prostate carcinoma cells. Flow cytometry was then carried out to detect whether Lv-shUBAP2L affects the cell cycle progression of prostate carcinoma cells. As shown in Fig. 6A and B, downregulation of UBAP2L induced $\mathrm{PC}-3$ cell accumulation in the $\mathrm{S}$ phase compared with the control cells. The percentage of cells in the $\mathrm{S}$ phase increased from $\sim 28.88 \%$ in the $\mathrm{Lv}$-shCon group to $38.33 \%$ in the Lv-shUBAP2L group. In addition, UBAP2L knockdown induced DU145 cell accumulation in the $\mathrm{G}_{2} / \mathrm{M}$ phase compared with the control cells (Fig. $6 \mathrm{C}$ and $\mathrm{D}$ ). The percentage of cells in the $\mathrm{G}_{2} / \mathrm{M}$ phase increased from $7.50 \%$ in the Lv-shCon group to $10.89 \%$ in the Lv-shUBAP2L group. The cell population in the $\mathrm{G}_{0} / \mathrm{G}_{1}$ phase was significantly decreased in both PC-3 and DU145 cells following UBAP2L knockdown. These results indicate that UBAP2L depletion inhibited cell proliferation by inducing cell cycle arrest.

Knockdown of UBAP2L inhibits the migration of prostate carcinoma cells. Cell migration is a critical step that occurs during cancer progression. We next determined the effect of UBAP2L knockdown in regulating prostate carcinoma cell 
$\mathbf{A}$

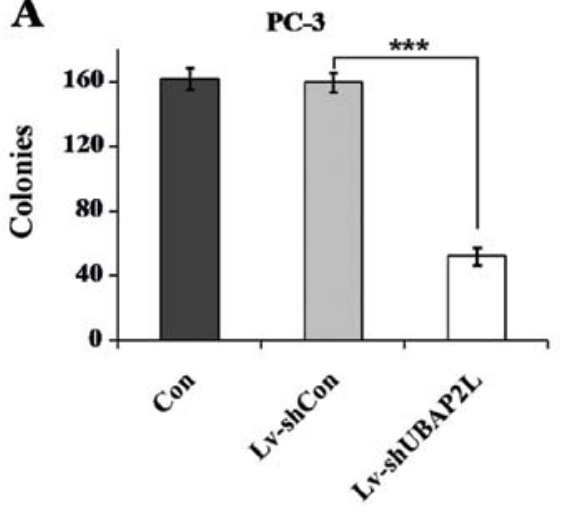

C

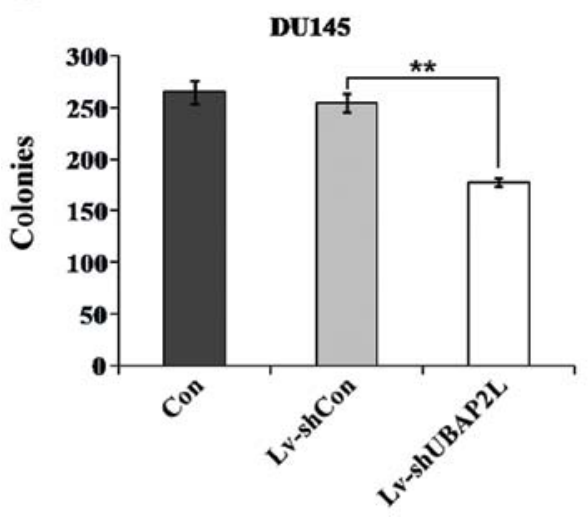

B
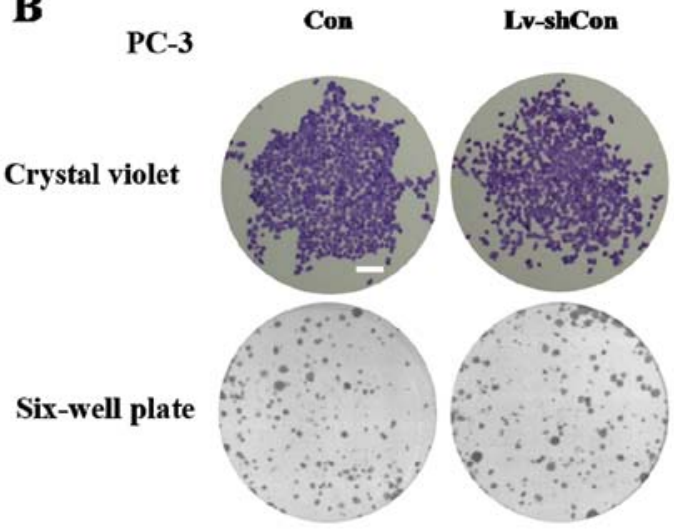

Lv-shUBAP2L

D

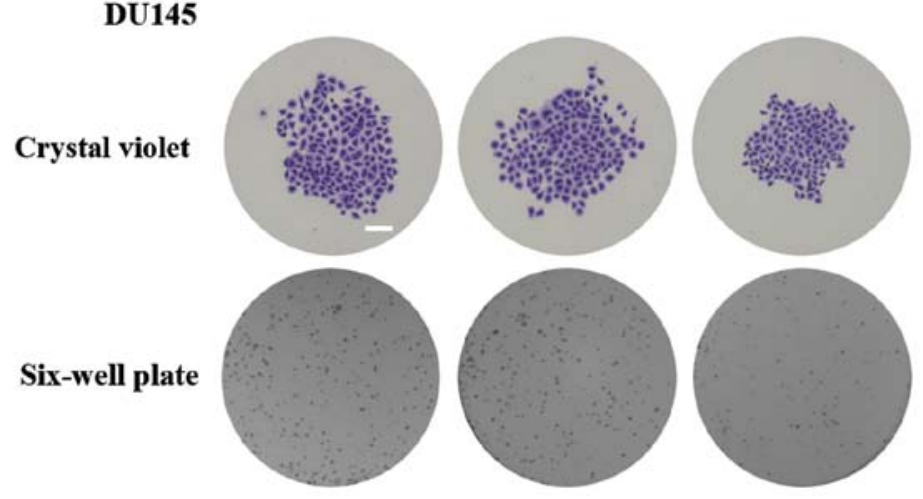

Figure 5. Knockdown of UBAP2L inhibits the colony formation of prostate carcinoma cells. Statistical analysis of the colony numbers of (A) PC-3 and (C) DU145 cells. Data represent the mean \pm SD of three independent experiments. ${ }^{* *} \mathrm{P}<0.01,{ }^{* * * *} \mathrm{P}<0.001$, compared with Lv-shCon. Representative images of colonies formed in (B) PC-3 and (D) DU145 cells under light microscopy. Scale bar, $125 \mu \mathrm{m}$.

$\mathbf{A}$

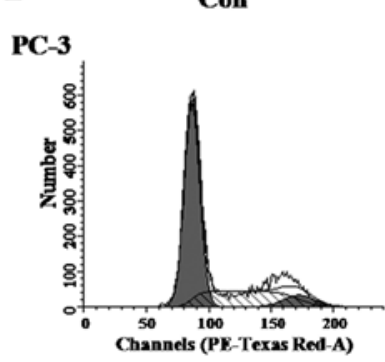

C

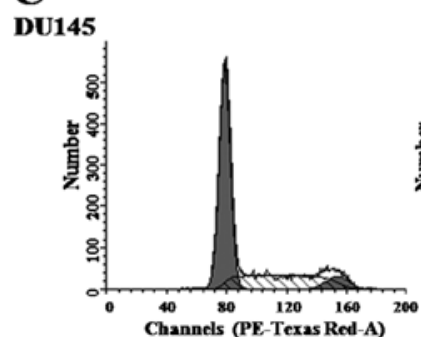

Lv-shCon
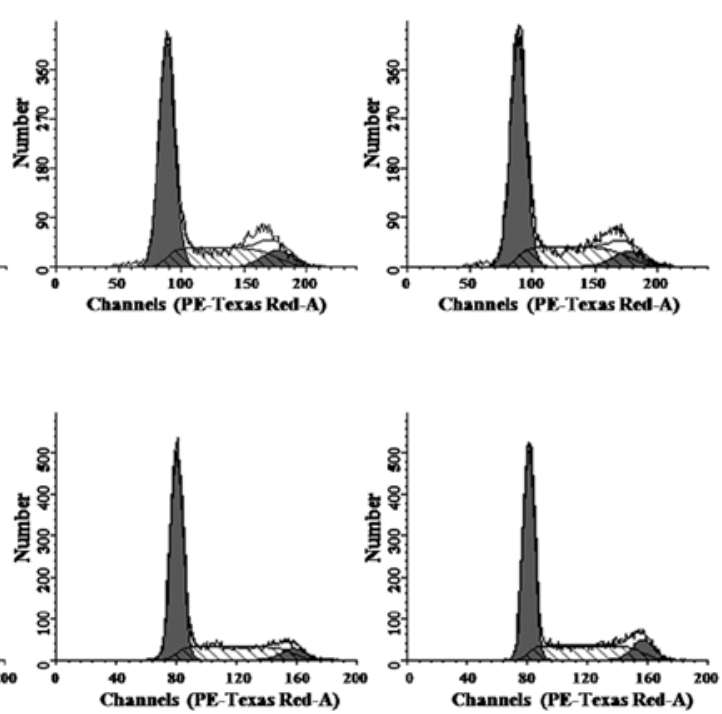
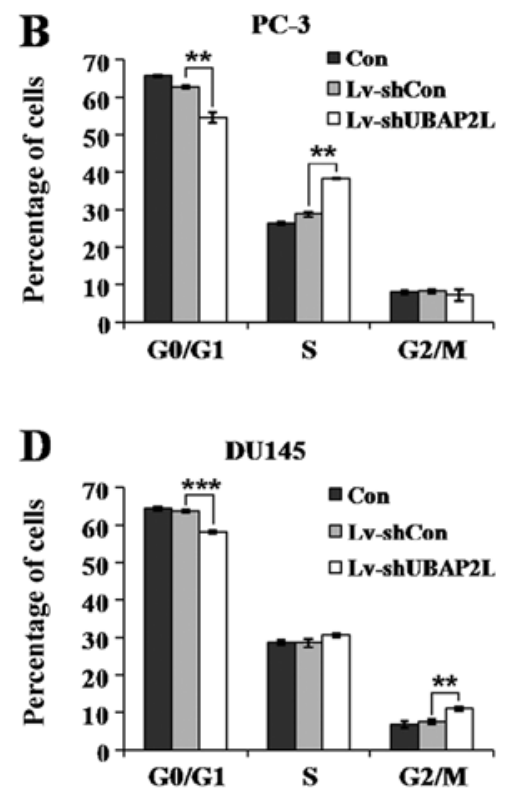

Figure 6. Knockdown of UBAP2L leads to cell cycle arrest. Fluorescence-activated cell sorting analysis of the cell cycle distribution in (A) PC-3 and (C) DU145 cells after lentivirus infection. The percentages of (B) PC-3 and (D) DU145 cells in G0/G1, S and G2/M phases. Data represent the mean \pm SD of three independent experiments. ${ }^{* *} \mathrm{P}<0.01,{ }^{* * * *} \mathrm{P}<0.001$, compared with $\mathrm{Lv}$-shCon.

migration by using Transwell assay (Fig. 7A and D). UBAP2L silencing markedly inhibited the migration of PC-3 and DU145 cells compared with the control cells. Fewer cells in the
Lv-shUBAP2L groups $(285.7 \pm 15.2$ PC-3 cells and $53.5 \pm 14.0$ DU145 cells) migrated into the lower filter relative to cells in the Lv-shCon groups (441.3 \pm 3.6 PC-3 cells and 108.0 \pm 2.8 DU145 
A

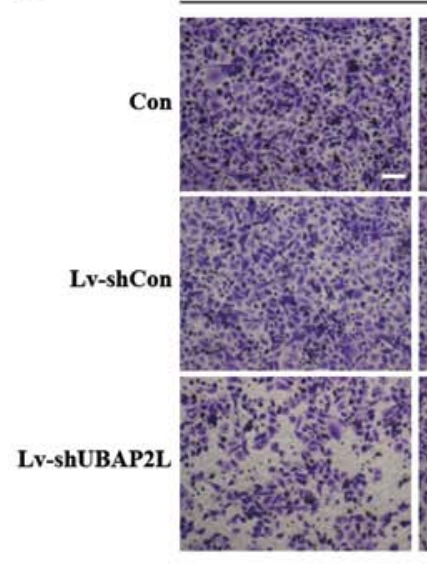

B

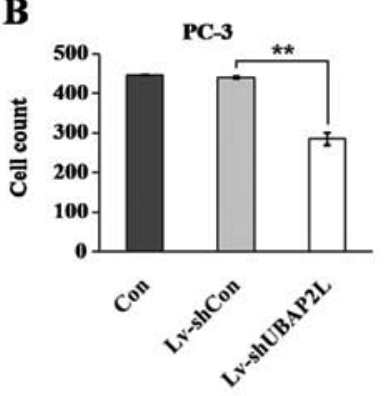

PC-3

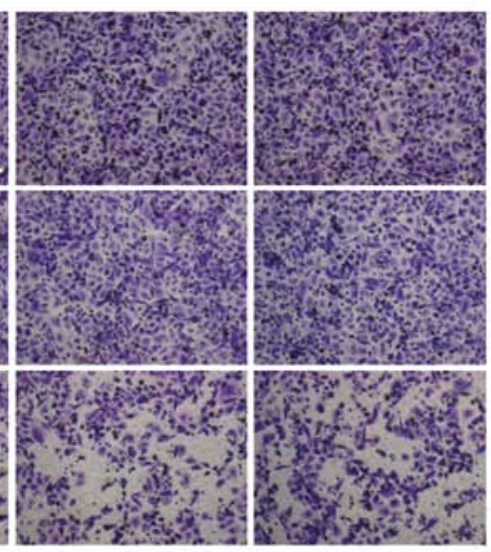

C

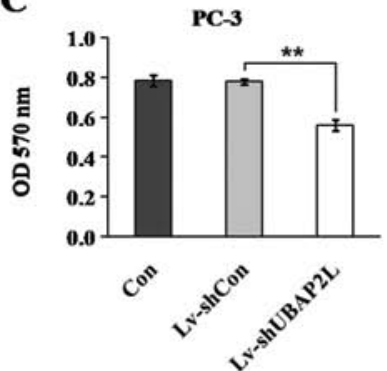

D

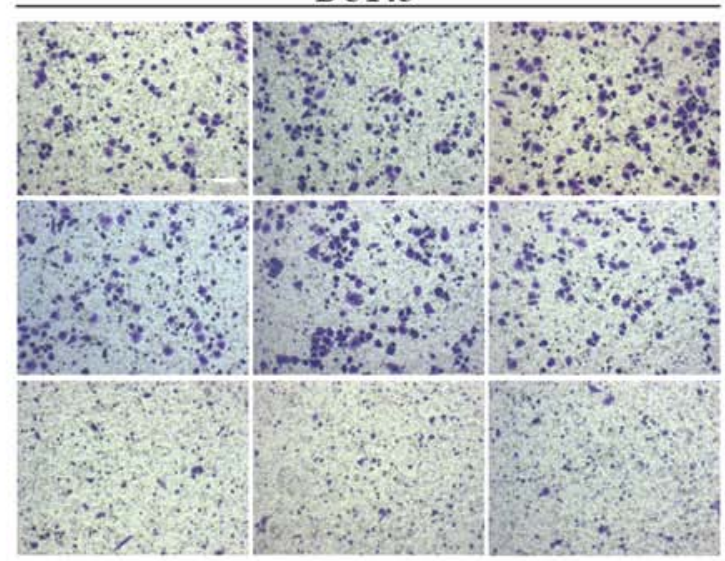

$\mathbf{E}$

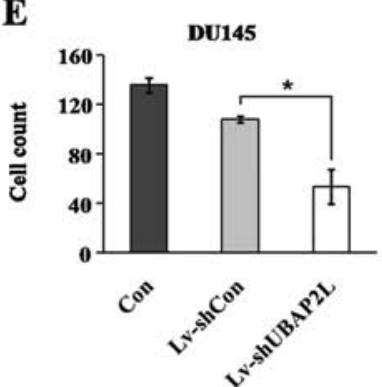

Figure 7. Knockdown of UBAP2L inhibits the migration of prostate carcinoma cells. Representative images of (A) PC-3 and (D) DU145 cells that migrated through Transwell membrane. Scale bar, $50 \mu \mathrm{m}$. Cell count of the migrated (B) PC-3 and (E) DU145 cells. Quantitative analysis of the migrated (C) PC-3 and (F) DU145 cells by destaining and reading optical density at $570 \mathrm{~nm}$. Data represent the mean $\pm \mathrm{SD}$ of three independent experiments. ${ }^{*} \mathrm{P}<0.05,{ }^{* *} \mathrm{P}<0.01$, compared with Lv-shCon.

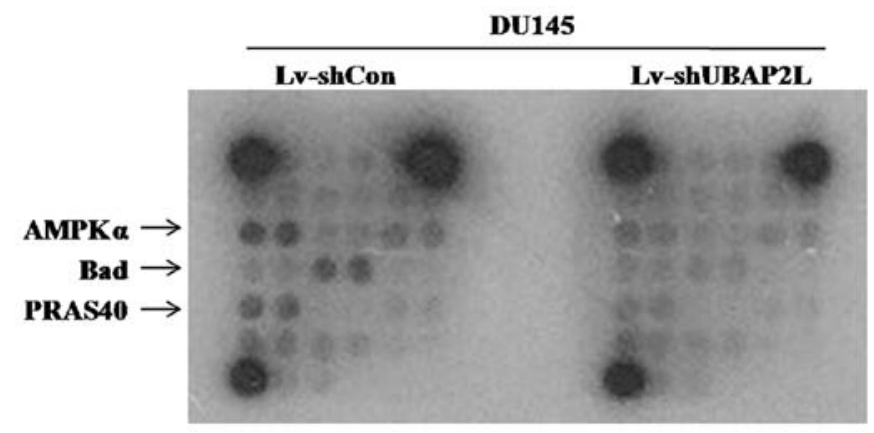

Figure 8. Knockdown of UBAP2L suppresses activation of AMPK $\alpha$, Bad and PRAS40. Intracellular signaling array was performed after Lv-shUBAP2L infection.

cells) (Fig. 7B and E). Moreover, the crystal violet staining intensity was significantly lower in the Lv-shUBAP2L groups than in the Lv-shCon and non-infected groups (Fig. 7C and F). The results suggest that UBAP2L is important for cell migration and may be a key player in prostate cancer metastasis.

Knockdown of UBAP2L blocks AMPKa, Bad and PRAS40 signaling pathways. To investigate the regulatory mechanism of UBAP2L in the tumorigenesis and metastasis of prostate carcinoma, multiple signaling pathways were analyzed in DU145 cells after UBAP2L knockdown using PathScan Intracellular Signaling Array kit. Compared with the Lv-shCon group, the amounts of phosphorylated AMP-activated protein kinase $\alpha(\mathrm{AMPK} \alpha)$, Bad and PRAS40 were obviously reduced in the Lv-shUBAP2L group (Fig. 8). Previous studies suggest that the AMPK $\alpha$, Bad and PRAS40 signaling pathways are crucial for carcinoma cell proliferation and cell cycle control (10-12). Our data revealed that UBAP2L depletion induced proliferation inhibition and cell cycle arrest via the AMPK $\alpha$, Bad and PRAS40 signaling pathways in prostate cancer.

\section{Discussion}

A previous study suggested that UBAP2L may be a component of the ubiquitin-proteasome complex (9). Inhibition of the ubiquitin-proteasome system is a promising anticancer strategy, and several proteasome inhibitors have been approved to be used in the clinic (4-6). In the present study, we found that UBAP2L was highly expressed in different prostate carcinoma cell lines. Taking advantage of lentiviral-mediated $\mathrm{RNAi}$, we disrupted the expression of UBAP2L in vitro. We found that UBAP2L depletion inhibited the proliferation and colony formation of prostate carcinoma cells. Cell cycle analysis showed that knockdown of UBAP2L led to PC-3 cell cycle arrest in the S phase, and DU145 cell cycle arrest in the $\mathrm{G}_{2} / \mathrm{M}$ phase. This difference in cell cycle arrest could be explained by the specific cell type. Moreover, UBAP2L knockdown inhibited the migration of prostate carcinoma cells. To investigate the molecular mechanisms of induced growth and migration inhibition following UBAP2L depletion, 
cell cycle-related signaling pathways were further analyzed using Pathscan Intracellular Signaling Array Kit. We found that UBAP2L depletion obviously inhibited the activation of AMPK $\alpha$, Bad and PRAS40.

AMPK, a serine-threonine kinase, is a heterotrimeric complex of catalytic $\alpha$-subunits and regulatory $\beta$ - and $\gamma$-subunits with multiple isoforms (13). AMPK is an energy sensor that is activated by phosphorylation at Thr172 in response to elevated AMP levels. AMPK regulates fatty acid metabolism, as well as modulates protein synthesis and cell growth. Although many studies support the tumor-suppressive role of AMPK, emerging evidence suggests that the metabolic checkpoint function of AMPK might be overridden by stress or oncogenic signals so that tumor cells use AMPK activation as a survival strategy to gain a growth advantage (10). These findings underscore the complexity in the cellular function of AMPK in maintaining energy homeostasis under physiological versus pathological conditions. Previously, Smith et al demonstrated that increased BAD expression promotes prostate cancer cell proliferation (12). The BAD phosphorylation status plays a major role in apoptosis regulation by serving as a convergence point of several anti-apoptotic signaling pathways, including constitutively active PI3K (14). BAD phosphorylation at serines 112 and 136 (based on the mouse sequence) $(15,16)$, facilitates interaction with 14-3-3 chaperones, whereas phosphorylation at S155 within the BH3 domain disrupts binding to BCL-XL or BCL-2 (17). As a result, phosphorylation inactivates the pro-apoptotic function of BAD by preventing interaction with BCL-2 and BCL-XL. PRAS40 is an mTOR binding protein that has complex effects on cell metabolism. PRAS40 regulates protein synthesis and the cell cycle (11). Phosphorylation of PRAS40 at Thr246 by Akt relieves PRAS40 inhibition of TORC1 (18). Our results suggest that UBAP2L may promote prostate carcinoma cell growth through activation of AMPK $\alpha$, Bad and PRAS40, which induce cell proliferation and cell cycle transition. However, the mechanisms involved in the regulation of the AMPK $\alpha$, Bad and PRAS40 signaling pathways by UBAP2L remain unknown. One explanation is that, as a potential component of proteasome, UBAP2L might directly regulate the degradation of AMPKa, Bad and PRAS40. Another possibility is that UBAP2L may regulates the expression of several other proteins, which regulate the phosphorylation of AMPK $\alpha$, Bad and PRAS40. Collectively, UBAP2L may mediate tumorigenesis and metastasis via regulation of the AMPK $\alpha$, Bad and PRAS40 signaling pathways.

In conclusion, we demonstrated for the first time that UBAP2L is crucial for the growth and migration of prostate carcinoma cells. UBAP2L silencing by RNAi may be a promising novel therapeutic method for prostate cancer.

\section{References}

1. Baade PD, Youlden DR and Krnjacki LJ: International epidemiology of prostate cancer: geographical distribution and secular trends. Mol Nutr Food Res 53: 171-184, 2009.

2. McGarrity GJ, Hoyah G, Winemiller A, et al: Patient monitoring and follow-up in lentiviral clinical trials. J Gene Med 15: 78-82, 2013.

3. Chen FZ and Zhao XK: Ubiquitin-proteasome pathway and prostate cancer. Onkologie 36: 592-596, 2013.

4. Iqbal M, Messina McLaughlin PA, Dunn D, et al: Proteasome inhibitors for cancer therapy. Bioorg Med Chem 20: 2362-2368, 2012.

5. Crawford LJ, Walker B and Irvine AE: Proteasome inhibitors in cancer therapy. J Cell Commun Signal 5: 101-110, 2011.

6. Rastogi N and Mishra DP: Therapeutic targeting of cancer cell cycle using proteasome inhibitors. Cell Div 7: 26, 2012.

7. San-Miguel JF, Richardson PG, Gunther A, et al: Phase Ib study of panobinostat and bortezomib in relapsed or relapsed and refractory multiple myeloma. J Clin Oncol 31: 3696-3703, 2013.

8. O'Brien ME, Gaafar RM, Popat S, et al: Phase II study of first-line bortezomib and cisplatin in malignant pleural mesothelioma and prospective validation of progression free survival rate as a primary end-point for mesothelioma clinical trials (European Organisation for Research and Treatment of Cancer 08052). Eur J Cancer 49: 2815-2822, 2013.

9. Wilde IB, Brack M, Winget JM and Mayor T: Proteomic characterization of aggregating proteins after the inhibition of the ubiquitin proteasome system. J Proteome Res 10: 1062-1072, 2011 .

10. Chuang HC, Chou CC, Kulp SK and Chen CS: AMPK as a potential anticancer target - friend or foe? Curr Pharm Des 20: 2607-2618, 2013.

11. Kazi AA and Lang CH: PRAS40 regulates protein synthesis and cell cycle in C2C12 myoblasts. Mol Med 16: 359-371, 2010.

12. Smith AJ, Karpova Y, D'Agostino R Jr, Willingham M and Kulik $\mathrm{G}$ : Expression of the Bcl-2 protein BAD promotes prostate cancer growth. PLoS One 4: e6224, 2009.

13. Li J, Coven DL, Miller EJ, et al: Activation of AMPK alpha- and gamma-isoform complexes in the intact ischemic rat heart. Am J Physiol Heart Circ Physiol 291: H1927-H1934, 2006.

14. Sastry KS, Smith AJ, Karpova Y, Datta SR and Kulik G: Diverse antiapoptotic signaling pathways activated by vasoactive intestinal polypeptide, epidermal growth factor, and phosphatidylinositol 3-kinase in prostate cancer cells converge on BAD. J Biol Chem 281: 20891-20901, 2006.

15. Datta SR, Katsov A, Hu L, et al: 14-3-3 proteins and survival kinases cooperate to inactivate $\mathrm{BAD}$ by $\mathrm{BH} 3$ domain phosphorylation. Mol Cell 6: 41-51, 2000.

16. Fang X, Yu S, Eder A, et al: Regulation of BAD phosphorylation at serine 112 by the Ras-mitogen-activated protein kinase pathway. Oncogene 18: 6635-6640, 1999.

17. Tan Y, Demeter MR, Ruan H and Comb MJ: BAD Ser-155 phosphorylation regulates BAD/Bcl-XL interaction and cell survival. J Biol Chem 275: 25865-25869, 2000.

18. Sancak Y, Thoreen CC, Peterson TR, et al: PRAS40 is an insulin-regulated inhibitor of the mTORC1 protein kinase. Mol Cell 25: 903-915, 2007. 